\title{
Actively modulated propagation of electromagnetic wave in hybrid metasurfaces containing graphene
}

\author{
Jiameng Nan, Ruisheng Yang, Jing Xu, Quanhong Fu, Fuli Zhang, and Yuancheng Fan ${ }^{*}$ \\ Key Laboratory of Light Field Manipulation and Information Perception, Ministry of Industry and Information Technology and \\ School of Physical Science and Technology, Northwestern Polytechnical University, Xi'an 710129, PR China
}

Received: 10 November 2020 / Accepted: 16 December 2020

\begin{abstract}
Here we present the actively modulated transportation of electromagnetic wave through hybrid metasurfaces containing graphene. The hybrid metasurfaces are composed of patterned metallic layers of extraordinary transmission and backed with graphene-sandwich layers. With the designed metallic layer with perforated structure, we demonstrated effective modulation on the on-resonance transmission amplitude by increasing the bias voltage from 0 to $4 \mathrm{~V}$ to electrically tune the Fermi level as well as the sheet resistance of the graphene-sandwich structure. We also found that the modulation depth can be further improved by properly designing the perforated metallic structure. By change the geometry from cut-wire structure to the "butterfly"like pattern we preliminarily achieved $19.2 \%$ improvement on the on-resonance transmission modulation. The measured transmittances of the active metasurfaces show good agreement with the numerical simulations with fitted graphene sheet resistances. The hybrid metasurfaces presented in this work may be deployed in a wide range of applications based on active electromagnetic or optical modulations.
\end{abstract}

Keywords: Graphene metasurface / extraordinary transmission / modulator / conductivity

\section{Introduction}

Metamaterials are artificially engineered subwavelength micro/nano-structures with novel optical properties hard to be found in natural materials [1-5]. Since their optical properties of can be arbitrarily and rationally designed by controlling the geometries of the structures, metamaterial and its two-dimensional (2D) counterpart, that is, the metasurface [6-11] have been proved to be a promising route for subwavelength optics or metaoptics based on metadevices [12]. In contrast to three-dimensional (3D) bulk metamaterials, metasurfaces with periodically arranged simple planar structures on $2 \mathrm{D}$ surfaces have been implanted for fully controlling of the state (amplitude, frequency, polarization, and phase) of light [13-17]. Although it has been proved that the metasurface is very powerful for manipulating light with enhanced interactions. The narrow operation band of metasurface may obstacle it to practical application due to the resonant nature of the composed structures.

Graphene stimulated and led the research upsurge in two-dimensional materials $[18,19]$ since its first exfoliation from graphite by Geim and Novoselov [20,21]. In photonics and optoelectronics, it has also been widely investigated for

\footnotetext{
* e-mail: phyfan@nwpu.edu.cn
}

potential applications based on the enabled stronger binding of surface plasmon polaritons [22-25] and the ultra-wideband tunability in optical response through electrostatic field, magnetic field, or chemical doping.

The wideband optical tunability of graphene provides the opportunity for realizing frequency-agile responses in metasurfaces containing the $2 \mathrm{D}$ sheet [26-28]. There have been numerous reports on scientific advances in graphene based metamaterials or metasurfaces especially in optical domain [29-31]. And some progresses have also been made with large scale graphene for lower frequency applications such as modulators, absorbers, and switches [32-44]. In this paper, we propose and demonstrate a kind hybrid metasurface based modulator in a more challenging case, i.e. the active modulation of transmitted wave since most of the works in microwave band were focused on the modulation of reflection $[45,46]$. The hybrid metasurfaces are composed of perforated metallic layers studied for extraordinary transmission [47-50] and backed with graphene-electrolyte-graphene sandwich structures. The extraordinary transmission structure was employed for the purpose of high modulation of transmittance in which the modulation depth is mainly limited by the conductivity range of the used graphene. Effective modulation on the onresonance transmission amplitude was demonstrated by increasing the bias voltage from 0 to $4 \mathrm{~V}$ to electrically tune the Fermi level as well as the sheet resistance of the 


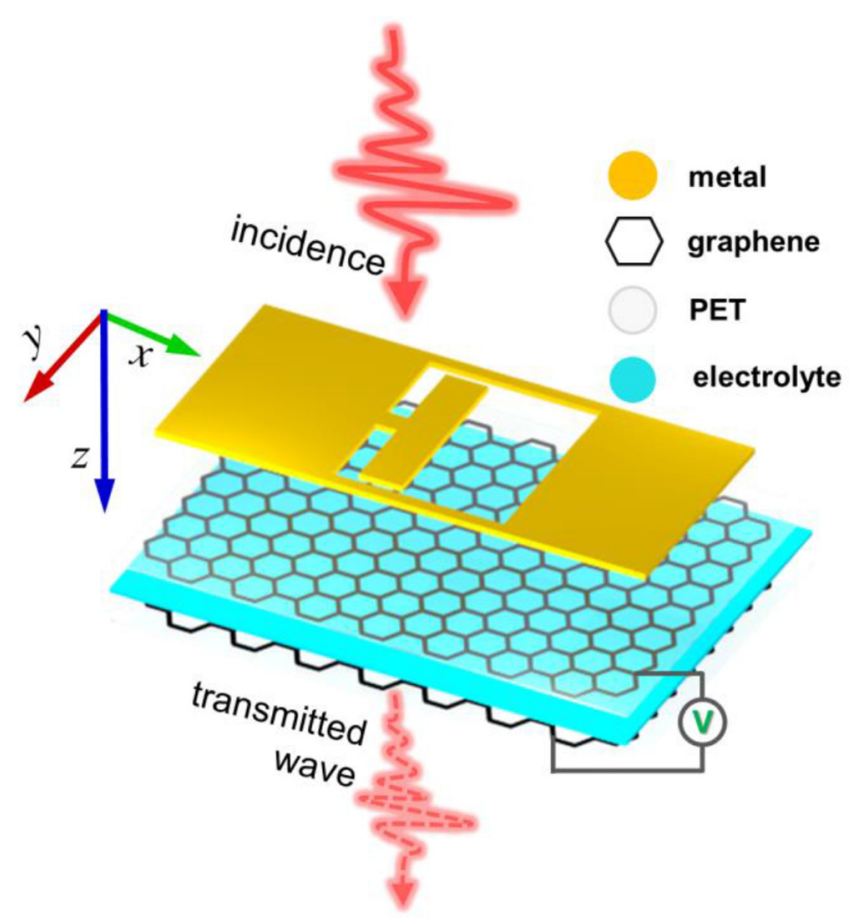

Fig. 1. Schematic illustration of the hybrid metasurfaces consisting of the patterned metallic layer and the electrically biased graphene sandwich structure.

graphene-sandwich structure. We also found that the modulation depth can be further improved by properly designing the perforated metallic structure.

\section{Results and discussion}

Figure 1 schematically shows the proposed hybrid metasurface composed of graphene-sandwich layers and perforated metallic structure, which is capable of dynamically manipulating extraordinary transmission under different bias voltages. In our design, double graphene monolayers (serve as graphene electrodes with dimension of $40 \times$ $20 \mathrm{~mm}^{2}$ ) are attached to both side of a $50 \mu \mathrm{m}$-thick diaphragm paper (serve as an electrolyte layer) which is entirely soaked in ionic liquid ([DEME] [TFSI]) and is of the same size as the graphene layers, forming a graphenesandwich structure. The graphene-sandwich layers are adhered to a $125 \mu \mathrm{m}$-thick Polyethylene terephthalate (PET) substrate. After applying the bias voltage, the graphene electrodes will polarize the electrolyte and form ionic bilayers of opposite polarity on graphene electrodes, which can generate the vertical bias. Therefore, the positive and negative ions will move and accumulate on the graphene electrodes, serving as a supercapacitor. The carrier concentration as well as the graphene's Fermi level can be effectively modulated through continuously controlling the bias voltage applied on the graphene electrodes. To electrically manipulate the extraordinary transmission, the metallic layer with perforated structure is then located on the top of the graphene-sandwich layers, forming a hybrid metasurface. The metallic pattern is $35 \mu \mathrm{m}$-thick copper on a $1 \mathrm{~mm}$ thick Teflon substrate and is fabricated through the standard printed circuit board (PCB) technology. The Teflon substrate was cut to dimension of $22.86 \times 10.16 \mathrm{~mm}^{2}$ corresponding to the cross-section of a standard waveguide WR90. In our simulations, the boundary conditions in the $\mathrm{x}$ - and $\mathrm{y}$-directions are set to the electrical boundaries, and the z-direction is set to the open boundary. In our experiments, the scattering parameters of the hybrid metasurface are measured inside a standard waveguide of WR90, where the $\mathrm{TE}_{10}$ mode with an electric field polarizing along the $\mathrm{y}$ direction is normally incident on the hybrid metasurface.

Since the wavelength in the microwave regime is much larger than the thickness of the graphene, the graphene in our design is modeled as a surface without thickness whose complex surface conductivity is $\sigma_{g}$. The Kubo formula is always used to characterize the conductivity of graphene [51], which includes two parts: intraband item and interband item. In the microwave band, the intraband contribution of graphene has a much greater influence than the interband item in terms of the conductivity, the interband item can be ignored, and the conductivity can be simplified as $[52,53]$ :

$$
\sigma_{g}=\sigma_{\text {intra }}=\frac{i 2 e^{2} K_{B} T}{\pi \hbar^{2}\left(\omega+i \tau^{-1}\right)} \ln \left[2 \cosh \left(\frac{E_{F}}{2 K_{B} T}\right)\right]
$$

where $e$ is the electron charge, and $K_{B}$ and $\hbar$ are the Boltzmann constant and the reduced Planck constant, respectively. $T$ is the room temperature. $\tau$ is the relaxation time and $E_{F}$ is the Fermi level. Furthermore, the microwave frequency range we studied is rather narrow, in that the surface conductivity or resistance can be modeled as a constant in comparison with experimental results.

To confirm that the graphene-sandwich layers can be efficiently modulated by the bias voltage, we firstly measured the transmission characteristics of a graphenesandwich sample under different voltages. Then we compared the experimental results with the simulation results to conclude the relationship between the impedance of the graphene-sandwich layers and the bias voltage (Fig. 2). First, a DC voltage is applied to the graphene electrodes of the sandwiched layers. And then the graphene-sandwich layer with the voltage is placed in the middle of the WR90 waveguide. The scattering parameters of the sample are measured by a vector network analyzer (Anritsu). The sheet resistances of the graphene-sandwich layers under different bias voltages from 0 to $4 \mathrm{~V}$ are shown in Figure 2. When there is no voltage applied, the sheet resistance of the graphenesandwich layers reaches up to $1300 \Omega \mathrm{sq}^{-1}$. As the voltage gradually increases, the resistance gradually decreases and then tends to be flat. The resistance is approximately $300 \Omega \mathrm{sq}^{-1}$ with the bias voltage increased to $4 \mathrm{~V}$. Thus, we proved that the bias voltage can efficiently and conveniently tune the electromagnetic parameters of the graphene-sandwich layers. 
We first designed the metallic layer with perforated structure composed of a cut-wire inside a hole (see inset in Fig. 3a) to achieve a high on-resonance transmission in the measured frequency range. The cut-wire structure could firstly localize the incident waves at a subwavelength scale and then couple with the hole and radiate toward forward direction to realize the extraordinary transmission. Experimental and simulation results show that a high transmission of $91 \%$ can be realized due to the extraordinary transmission (not shown here). We then placed the perforated metallic structure on the top of the graphenesandwich layers for a hybrid metasurface (see Fig. 1) and studied how the transmission spectra of the hybrid metasurface changed under different bias voltages under illumination. The transmission spectra of the hybrid metasurface with metallic cut-wire under different resistances and voltages are shown in Figures 3a and 3b, respectively. A fabricated sample is shown in the inset of Figure 3b. The measured results show good agreement with the simulated results. We can observe that the overall

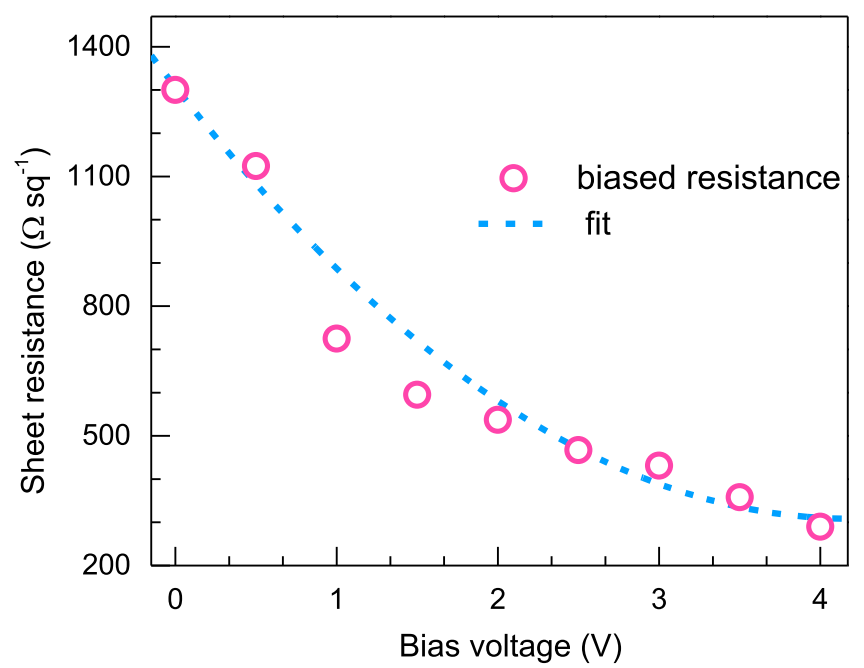

Fig. 2. Electrically biased sheet resistance of the graphenesandwich structure under the different bias voltage. transmission decreases with the increased bias voltage. In our experiment, when the voltage increases from 1.0 to $1.5 \mathrm{~V}$, the transmittance decreases from $47.7 \%$ to $37.9 \%$ at on-resonance frequency, which shows a large drop off of $9.8 \%$. With further increasing the voltage, the transmittance spectrum slowly changes. For example, as the voltage changes from 3.5 to $4.0 \mathrm{~V}$, the transmission at on-resonance frequency decreases from $24.4 \%$ to $20.9 \%$, showing only a $3.5 \%$ drop off. The significant drop from 1.0 to $1.5 \mathrm{~V}$ is due to the possible introduction of chemical doping in the preparation or transfer of the graphene. The two graphene have different Fermi levels, so the charge neutral point (CNP) of the graphene is shifted. At the CNP the graphene resistance reaches the maximum value, while the capacitance reaches the minimum value. This trend is consistent with the results in Figure 2. The transmission of the perforated structure alone can reach up to $91 \%$ (not shown here). However, the transmission is only $59.6 \%$, showing a $31.4 \%$ drop off when the graphene-sandwich layer was introduced. To reveal the energy losses in the metasurface, we calculate the energy loss distribution of the hybrid metasurface at on-resonance frequency $10.3 \mathrm{GHz}$. As shown in Figure 3c, the energy loss of the hybrid metasurface mainly occurs in the graphene-sandwich layers, in particular in the graphene layers.

For metasurfaces, one can design and optimize the geometry, size and electromagnetic parameters of their building blocks to improve the performance. To reach a higher extraordinary transmission and modulation depth, we hereafter propose another metallic layer with perforated structure that has a "butterfly"-like pattern. This symmetric "butterfly"-like configuration can be better coupled to the symmetrical incident wave in waveguide and then radiates out, resulting a higher transmission as well as the modulation depth.

The "butterfly"-like structure is schematically shown in the inset of Figure 4a, and a fabricated sample is shown in the inset of Figure $4 \mathrm{~b}$. Same as the first cut-wire assisted graphene hybrid metasurface, we placed the perforated "butterfly"-like metallic structure on the top of the proposed graphene-sandwich layers. The calculated and
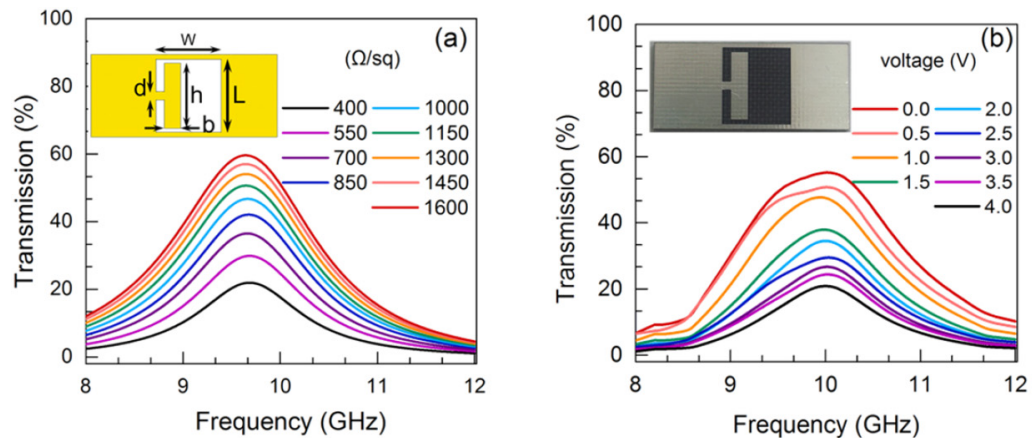

(c)

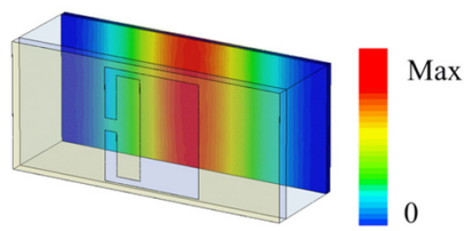

Fig. 3. (a) Calculated and (b) measured transmission spectra of the hybrid metasurface with metallic cut-wire structure by changing the sheet resistance from 400 to $1600 \Omega \mathrm{sq}^{-1}$, the external bias voltage changes from 0 to $4 \mathrm{~V}$ in experiments. The inset in (a) shows the schematic of the cut-wire structure and the inset in (b) shows a fabricated sample. The geometric parameters are: $\mathrm{L}=9 \mathrm{~mm}, \mathrm{~W}=8 \mathrm{~mm}$, $\mathrm{h}=8 \mathrm{~mm}, \mathrm{~b}=2 \mathrm{~mm}$, and $\mathrm{d}=1 \mathrm{~mm}$. (c) Energy loss distribution of the hybrid metasurface at frequency $10.3 \mathrm{GHz}$ with a sheet resistances of $1600 \Omega \mathrm{sq}^{-1}$. 

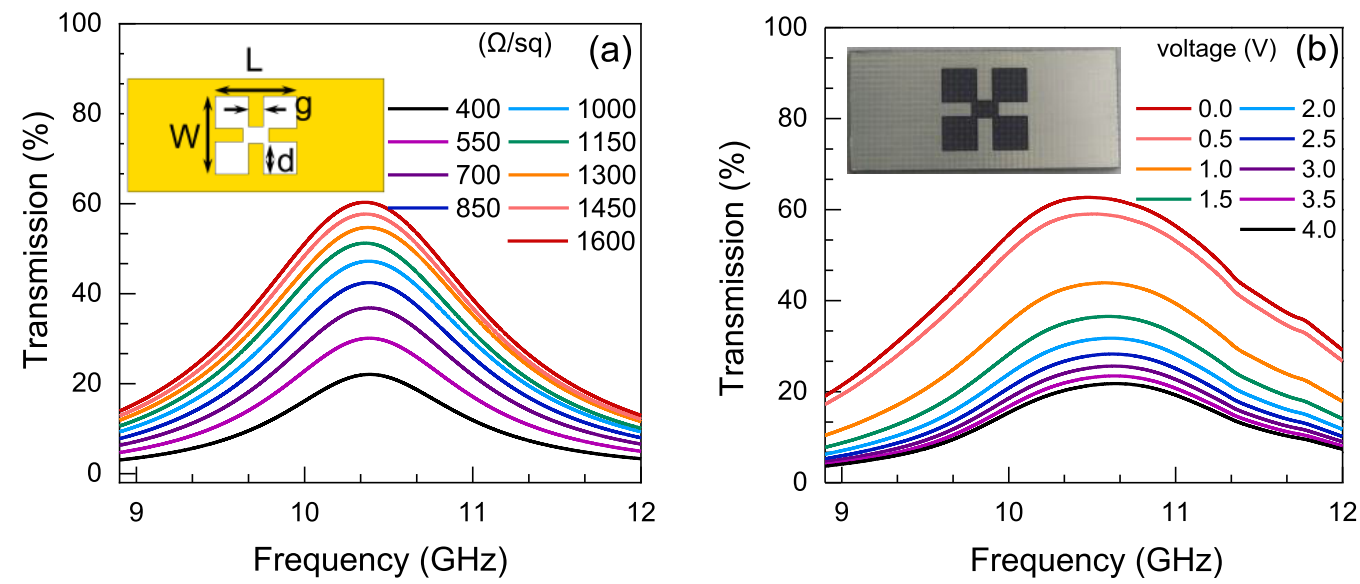

Fig. 4. (a) Calculated and (b) measured transmission spectra of the hybrid metasurface with metallic "butterfly"-like pattern by changing the sheet resistance from 400 to $1600 \Omega \mathrm{sq}^{-1}$, the external bias voltage changes from 0 to $4 \mathrm{~V}$ in experiments. The inset in (a) shows the schematic of the "butterfly"-like structure and the inset in (b) shows a fabricated sample. The geometric parameters are: $\mathrm{L}=7.3 \mathrm{~mm}, \mathrm{~W}=7 \mathrm{~mm}, \mathrm{~g}=1.3 \mathrm{~mm}$, and $\mathrm{d}=2.75 \mathrm{~mm}$.

measured transmission spectra of this hybrid metasurface under different resistances and different voltages are shown in Figure 4. The measured results are also in good agreement with the simulated results. The measured transmission (see Fig. 4b) decreases from $59.0 \%$ to $43.9 \%$ at on-resonance frequency with the bias voltage changing from 0.5 to $1.0 \mathrm{~V}$, showing a larger drop off of $15.1 \%$. As the voltage further increases, the transmittance also slowly decreases. For example, as the voltage increases from 3.5 to $4.0 \mathrm{~V}$, the transmission drops from $23.5 \%$ to $21.8 \%$ at on-resonance frequency, showing only a $1.7 \%$ drop off. Theoretically, the sheet resistance of the graphenesandwich layers can be further decreased as the voltage continues to increase. However, in reality, the conductivity of the graphene can only reach to a finite value due to some factors such as the quality of graphene, the chemical window of ionic liquid and the constructed model, and the resulting transmission spectra cannot be further modulated.

To intuitively visualize the differences of the two hybrid metasurfaces, we here plot the on-resonance transmission with different bias voltages, as shown in Figure 5 . The transmission of both hybrid metasurfaces shows a downward trend with increased bias voltage. For the first hybrid metasurface with metallic cut-wire structure, the transmission drops from $55.2 \%$ to $20.9 \%$, while for the metallic "butterfly"-like pattern, it drops from $62.7 \%$ to $21.8 \%$, when the bias voltage increases from 0 to $4 \mathrm{~V}$. Obviously, for the second hybrid metasurface with "butterfly"-like metallic pattern, the modulation depth can be further improved, about $19.2 \%$ improvement on the on-resonance transmission modulation is achieved. We note that although our results were obtained in a waveguide system, the nearly same modulation performances can also be realized with periodic structures and large scale graphene samples in free space.

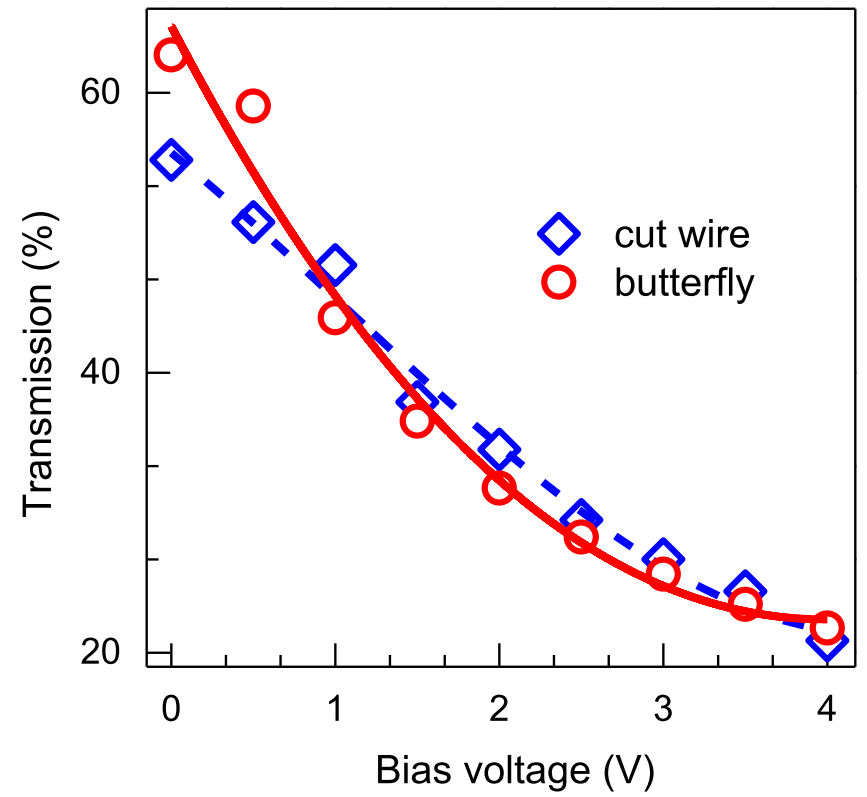

Fig. 5. The transmission at the on-resonance frequency with different bias voltages ranging from 0 to $4 \mathrm{~V}$ for the two hybrid metasurfaces.

\section{Conclusions}

In summary, we have demonstrated that hybrid metasurfaces composed of perforated metallic layers on the top of a graphene-sandwich structure for active modulation on the on-resonance transmission amplitude. It is found that the transmission of the hybrid metasurfaces can be achieved by increasing the bias voltage from 0 to $4 \mathrm{~V}$ to electrically tune the Fermi level as well as the sheet resistance of the graphene-sandwich structure. By chang- 
ing the geometry from cut-wire structure to the "butterfly"like pattern, a $19.2 \%$ improvement on the on-resonance transmission modulation is also realized. The proposed design strategy of the hybrid metasurfaces is promising for many applications based on active electromagnetic or optical modulations.

The authors would like to acknowledge financial support from Natural Science Foundation of China (NSFC) (Grants No. 12074314, 11674266, 61771402), NPU AoXiang New Star program, and Shaanxi Province Postdoctoral Science Foundation (No. 2018BSHEDZZ64).

\section{References}

1. D.R. Smith, J.B. Pendry, M.C.K. Wiltshire, Metamaterials and Negative Refractive Index, Science 305, 788 (2004)

2. S. Linden, C. Enkrich, M. Wegener, J.F. Zhou, T. Koschny, C.M. Soukoulis, Magnetic response of metamaterials at 100 terahertz, Science 306, 1351 (2004)

3. H.T. Chen, W.J. Padilla, J.M.O. Zide, A.C. Gossard, A.J. Taylor, R.D. Averitt, Active terahertz metamaterial devices, Nature 444, 597 (2006)

4. Q. Zhao, J. Zhou, F.L. Zhang, D. Lippens, Mie resonancebased dielectric metamaterials, Mater. Today 12, 60 (2009)

5. S. Jahani, Z. Jacob, All-dielectric metamaterials, Nat. Nanotechnol. 11, 23 (2016)

6. N.F. Yu, P. Genevet, M.A. Kats, F. Aieta, J.P. Tetienne, F. Capasso, Z. Gaburro, Light propagation with phase discontinuities: generalized laws of reflection and refraction, Science 334, 333 (2011)

7. S. Sun, Q. He, S. Xiao, Q. Xu, X. Li, L. Zhou, Gradient-index meta-surfaces as a bridge linking propagating waves and surface waves, Nat. Mater. 11, 426 (2012)

8. S.B. Glybovski, S.A. Tretyakov, P.A. Belov, Y.S. Kivshar, C. R. Simovski, Metasurfaces: from microwaves to visible, Phys. Rep. 634, 1 (2016)

9. F. Ding, A. Pors, S.I. Bozhevolnyi, Gradient metasurfaces: a review of fundamentals and applications, Rep. Prog. Phys. 81, 026401 (2017)

10. S. Wang, P.C. Wu, V.C. Su, Y.C. Lai, M.K. Chen, H.Y. Kuo, B.H. Chen, Y.H. Chen, T.T. Huang, J.H. Wang, R.M. Lin, C. H. Kuan, T. Li, Z. Wang, S. Zhu, D.P. Tsai, A broadband achromatic metalens in the visible, Nat. Nanotechnol. 13, $227(2018)$

11. A.M. Shaltout, V.M. Shalaev, M.L. Brongersma, Spatiotemporal light control with active metasurfaces, Science $\mathbf{3 6 4}$, eaat3100 (2019)

12. N.I. Zheludev, Y.S. Kivshar, From metamaterials to metadevices, Nat. Mater. 11, 917 (2012)

13. A.V. Kildishev, A. Boltasseva, V.M. Shalaev, Planar photonics with metasurfaces, Science 339, 1232009 (2013)

14. H. Cheng, Z. Liu, S. Chen, J. Tian, Emergent functionality and controllability in few-layer metasurfaces, Adv. Mater. 27, $5410(2015)$

15. H.-T. Chen, A.J. Taylor, N. Yu, A review of metasurfaces: physics and applications, Rep. Prog. Phys. 79, 076401 (2016)

16. S. Chen, Z. Li, W. Liu, H. Cheng, J. Tian, From singledimensional to multidimensional manipulation of optical waves with metasurfaces, Adv. Mater. 31, 1802458 (2019)
17. R.S. Yang, Q.H. Fu, Y.C. Fan, W.Q. Cai, K.P. Qiu, W.H. Zhang, F.L. Zhang, Active control of EIT-like response in a symmetry-broken metasurface with orthogonal electric dipolar resonators, Photonics Res. 7, 955 (2019)

18. S.Z. Butler, S.M. Hollen, L. Cao, Y. Cui, J.A. Gupta, H.R. Gutierrez, T.F. Heinz, S.S. Hong, J. Huang, A.F. Ismach, E. Johnston-Halperin, M. Kuno, V.V. Plashnitsa, R.D. Robinson, R.S. Ruoff, S. Salahuddin, J. Shan, L. Shi, M. G. Spencer, M. Terrones, W. Windl, J.E. Goldberger, Progress, challenges, and opportunities in two-dimensional materials beyond graphene, ACS Nano 7, 2898 (2013)

19. G. Fiori, F. Bonaccorso, G. Iannaccone, T. Palacios, D. Neumaier, A. Seabaugh, S.K. Banerjee, L. Colombo, Electronics based on two-dimensional materials, Nat. Nanotechnol. 9, 768 (2014)

20. K.S. Novoselov, A.K. Geim, S.V. Morozov, D. Jiang, Y. Zhang, S.V. Dubonos, I.V. Grigorieva, A.A. Firsov, Electric field effect in atomically thin carbon films, Science $\mathbf{3 0 6}$, $666(2004)$

21. A.K. Geim, K.S. Novoselov, The rise of graphene, Nat. Mater. 6, 183 (2007)

22. N.-H. Shen, M. Massaouti, M. Gokkavas, J.-M. Manceau, E. Ozbay, M. Kafesaki, T. Koschny, S. Tzortzakis, C.M. Soukoulis, Optically implemented broadband blueshift switch in the terahertz regime, Phys. Rev. Lett. 106 037403 (2011)

23. O. Balci, N. Kakenov, E. Karademir, S. Balci, S. Cakmakyapan, E.O. Polat, H. Caglayan, E. Özbay, C. Kocabas, Electrically switchable metadevices via graphene, Sci. Adv. 4 eaao1749 (2018)

24. Y. Fan, N.-H. Shen, F. Zhang, Q. Zhao, Z. Wei, P. Zhang, J. Dong, Q. Fu, H. Li, C.M. Soukoulis, Photoexcited graphene metasurfaces: significantly enhanced and tunable magnetic resonances, ACS Photonics 5, 1612 (2018)

25. W. Wang, Z. Song, Multipole plasmons in graphene nanoellipses, Physica B 530, 142 (2018)

26. M. Freitag, T. Low, F. Xia, P. Avouris, Photoconductivity of biased graphene, Nat. Photonics 7, 53 (2013)

27. S. Huang, C. Song, G. Zhang, H. Yan, Graphene plasmonics: physics and potential applications, Nanophotonics 6, 1191 (2017)

28. Y. Fan, N.H. Shen, F. Zhang, Q. Zhao, H. Wu, Q. Fu, Z. Wei, H. Li, C.M. Soukoulis, Graphene plasmonics: a platform for 2D optics, Adv. Opt. Mater. 7, 1800537 (2019)

29. H. Yan, T. Low, W. Zhu, Y. Wu, M. Freitag, X. Li, F. Guinea, P. Avouris, F. Xia, Damping pathways of mid-infrared plasmons in graphene nanostructures, Nat. Photonics 7, 394 (2013)

30. Y. Fan, N.-H. Shen, T. Koschny, C.M. Soukoulis, Tunable terahertz meta-surface with graphene cut-wires, ACS Photonics 2, 151 (2015)

31. F.J. Bezares, A. De Sanctis, J.R.M. Saavedra, A. Woessner, P. Alonso-Gonzalez, T. Amenabar, J. Chen, T.H. Bointon, S. Dai, M.M. Fogler, D.N. Basov, R. Hillenbrand, M.F. Craciun, F.J. Garcia de Abajo, S. Russo, F.H.L. Koppens, Intrinsic plasmon-phonon interactions in highly doped graphene: a near-field imaging study, Nano Lett. 17, 5908 (2017)

32. S.H. Lee, M. Choi, T.-T. Kim, S. Lee, M. Liu, X. Yin, H.K. Choi, S.S. Lee, C.-G. Choi, S.-Y. Choi, Switching terahertz waves with gate-controlled active graphene metamaterials, Nat. Mater. 11, 936 (2012) 
33. B. Sensale-Rodriguez, R. Yan, S. Rafique, M. Zhu, W. Li, X. Liang, D. Gundlach, V. Protasenko, M.M. Kelly, D. Jena, Extraordinary control of terahertz beam reflectance in graphene electro-absorption modulators, Nano Lett. 12, 4518 (2012)

34. P.-Y. Chen, J. Soric, Y.R. Padooru, H.M. Bernety, A.B. Yakovlev, A. Alu, Nanostructured graphene metasurface for tunable terahertz cloaking, New J. Phys. 15, 123029 (2013)

35. H. Nasari, M.S. Abrishamian, Magnetically tunable focusing in a graded index planar lens based on graphene, J. Opt. 16, $105502(2014)$

36. B. Wu, H.M. Tuncer, A. Katsounaros, W. Wu, M.T. Cole, K. Ying, L. Zhang, W.I. Milne, Y. Hao, Microwave absorption and radiation from large-area multilayer CVD graphene, Carbon 77, 814 (2014)

37. O. Balci, E.O. Polat, N. Kakenov, C. Kocabas, Grapheneenabled electrically switchable radar-absorbing surfaces, Nat. Commun. 6, 6628 (2015)

38. N. Kakenov, O. Balci, E.O. Polat, H. Altan, C. Kocabas, Broadband terahertz modulators using self-gated graphene capacitors, JOSA B 32, 1861 (2015)

39. N. Kakenov, O. Balci, T. Takan, V.A. Ozkan, H. Altan, C. Kocabas, Observation of gate-tunable coherent perfect absorption of terahertz radiation in graphene, ACS Photonics 3, 1531 (2016)

40. Y. Fan, L. Tu, F. Zhang, Q. Fu, Z. Zhang, Z. Wei, H. Li, Broadband terahertz absorption in graphene-embedded photonic crystals, Plasmonics 13, 1153 (2018)

41. N. Kakenov, M.S. Ergoktas, O. Balci, C. Kocabas, Graphene based terahertz phase modulators, 2D Mater. 5, 035018 (2018)

42. Y. Hu, T. Jiang, J. Zhou, H. Hao, H. Sun, H. Ouyang, M. Tong, Y. Tang, H. Li, J. You, Ultrafast terahertz frequency and phase tuning by all-optical molecularization of metasurfaces, Adv. Opt. Mater. 7, 1901050 (2019)
43. L. Liu, W. Liu, Z. Song, Ultra-broadband terahertz absorber based on a multilayer graphene metamaterial, J. Appl. Phys. 128, 093104 (2020)

44. M. Zhang, Z. Song, Terahertz bifunctional absorber based on a graphene-spacer-vanadium dioxide-spacer-metal configuration, Opt. Express 28, 11780 (2020)

45. O. Balci, N. Kakenov, C. Kocabas, Controlling phase of microwaves with active graphene surfaces, Appl. Phys. Lett. 110, 161102 (2017)

46. J. Zhang, H. Zhang, W. Yang, K. Chen, X. Wei, Y. Feng, R. Jin, W. Zhu, Dynamic scattering steering with graphenebased coding metamirror, Adv. Opt. Mater. 8, 2000683 (2020)

47. L. Martin-Moreno, F.J. Garcia-Vidal, H.J. Lezec, K.M. Pellerin, T. Thio, J.B. Pendry, T.W. Ebbesen, Theory of extraordinary optical transmission through subwavelength hole arrays, Phys. Rev. Lett. 86, 1114 (2001)

48. C. Genet, T. W. Ebbesen, Light in tiny holes, Nature 445, 39-46 (2007)

49. H. Liu, P. Lalanne, Microscopic theory of the extraordinary optical transmission, Nature 452, 728 (2008)

50. K. Aydin, A.O. Cakmak, L. Sahin, Z. Li, F. Bilotti, L. Vegni, E. Ozbay, Split-ring-resonator-coupled enhanced transmission through a single subwavelength aperture, Phys. Rev. Lett. 102, 013904 (2009)

51. V. Gusynin, S. Sharapov, J. Carbotte, Magneto-optical conductivity in graphene, J. Phys.: Condens. Matter 19, $026222(2006)$

52. G.W. Hanson, Dyadic Green's functions and guided surface waves for a surface conductivity model of graphene, J. Appl. Phys. 103, 064302 (2008)

53. G.W. Hanson, Dyadic Green's functions for an anisotropic, non-local model of biased graphene, IEEE Trans. Antennas Propag. 56, 747 (2008)

Cite this article as: Jiameng Nan, Ruisheng Yang, Jing Xu, Quanhong Fu, Fuli Zhang, Yuancheng Fan, Actively modulated propagation of electromagnetic wave in hybrid metasurfaces containing graphene, EPJ Appl. Metamat. 7, 9 (2020) 